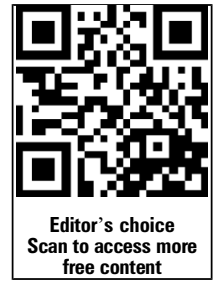
free content
- Additional material is published online only. To view please visit the journal online (http://dx.doi.org/10.1136/ neurintsurg-2014-011135).

${ }^{1}$ Case Western Reserve University School of Medicine, Cleveland, Ohio, USA 2Department of Epidemiology and Biostatistics, Case Western Reserve University, Cleveland, Ohio, USA

${ }^{3}$ Departments of Pulmonary, Critical Care, and Sleep Medicine, University Hospitals Case Medical Center, Cleveland, Ohio, USA ${ }^{4}$ Heart and Vascular Institute, Cleveland Clinic, Cleveland, Ohio, USA

${ }^{5}$ Department of Neurological Surgery, University Hospitals

Case Medical Center, Cleveland, Ohio, USA

\section{Correspondence to} Dr Nicholas Bambakidis, Department of Neurological Surgery, University Hospitals Case Medical Center, 11100 Euclid Avenue, Cleveland, OH 44106, USA Kimberly.Duvall@UHhospitals. org

Received 22 January 2014 Revised 5 April 2014 Accepted 7 April 2014 Published Online First 24 April 2014

\title{
Impact of age on 30-day postoperative outcome of surgery for ruptured and unruptured intracranial aneurysms
}

\author{
Nima Alan, ${ }^{1}$ Andreea Seicean, ${ }^{2}$ Sinziana Seicean, ${ }^{3,4}$ Warren R Selman, ${ }^{5}$ \\ Nicholas C Bambakidis ${ }^{5}$
}

\section{ABSTRACT}

Objective To assess in a retrospective analysis of a prospectively collected database, the impact of increased age on 30-day postoperative outcomes of surgery for intracranial aneurysms (ICAs).

Methods 721 adult patients who underwent surgery for ICA were identified in the 2006-2012 American College of Surgeons' National Surgical Quality Improvement Program. Baseline characteristics and 30day outcomes were stratified by age: $<50$ years $(n=221)$, $50-60$ years $(n=221)$, and $>60$ years $(n=266)$. Patients $<50$ and $50-60$ years old were propensity scorematched to those aged $>60$ years. Logistic regression was used to examine the relationship between increased age and surgical outcome.

Results In unadjusted analyses, age $<50$ years was associated with fewer postoperative complications (OR $=0.5,95 \% \mathrm{Cl} 0.3$ to 0.7 ) and lower mortality ( $O R=0.4,95 \% \mathrm{Cl} 0.2$ to 0.9 ) compared with those aged $>60$ years. Patients aged between 50 and 60 years were less likely to have complications ( $\mathrm{OR}=0.6,95 \% \mathrm{Cl} 0.4$ to 0.8 ) in unadjusted analyses. Upon propensity score matching, covariate balance was achieved for all age strata. In adjusted analyses, patients $<50$ years $(O R=0.4$, $95 \% \mathrm{Cl} 0.2$ to 0.7$)$ and $50-60$ years $(\mathrm{OR}=0.5,95 \% \mathrm{Cl}$ 0.3 to 0.8 ) of age continued to have fewer complications than those aged $>60$.

Conclusions Age $>60$ is independently associated with 30-day postoperative morbidity in patients undergoing surgery for ICA. The results of this study suggest age $>60$ should be considered an a priori risk factor in surgical management of ICA, regardless of associated comorbidities often associated with increased age.

\section{INTRODUCTION}

Intracranial aneurysms (ICAs) are common. Autopsy studies report aneurysms to be present in $5 \%$ of the population. ${ }^{1}$ Annually, $10-15$ per 100000 patients have an aneurysmal subarachnoid hemorrhage (SAH), a devastating condition that leads to death or severe neurological deficits in the majority of patients. ${ }^{2}$ Management of outcomes of ruptured and unruptured ICAs has improved over the years, but the impact of age on neurosurgical outcomes in this patient group remains uncertain. Numerous studies describe the impact of age on postoperative morbidity and mortality after surgical treatment of ICA, but the findings are inconclusive. $^{3-11}$ Most studies are limited owing to inadequate control for comorbidities that are often associated with advancing age but may have a confounding effect on surgical outcomes. Given the ageing population of the USA and increased incidence of ICA with age, ${ }^{12}$ it is important to elucidate the role of age in surgical management of ICA.

The aim of this study was to investigate the impact of increased age on the outcome of surgical management of ICAs. Using the 2006-2012 American College of Surgeons (ACS) National Surgical Quality Improvement Program (NSQIP), we compared the 30-day morbidity and mortality in patients $>60$ years old with those aged 50-60 and $<50$ years who underwent craniotomy for treatment of ICA.

\section{METHODS}

Database

Patients who underwent surgery for ICA during the 2006-2012 interval were identified in the ACS-NSQIP, which reports 30-day morbidity and mortality, with $95 \%$ success rate, for inpatient and outpatient surgeries from nearly 400 academic and non-academic hospitals nationwide. ${ }^{13}$ Data on randomly assigned consenting patients are collected prospectively by a trained surgical clinical reviewer in each participating institution. Data collection and inter-rater reliability auditing processes are highly reliable in NSQIP. ${ }^{14}$ Neurosurgical data collection started in 2006, with the most recent report released for 2012. Design, sampling strategy, variable definitions, and further description of the NSQIP database have been dealt with elsewhere. ${ }^{15} 16$

\section{Study population}

Using ICD-9 code 430 for SAH and 437.3 for unruptured cerebral aneurysm, we identified 962 patients, aged $\geq 18$ years, who had undergone surgery for treatment of ICA. We eliminated patients who had undergone surgery for treatment of arteriovenous malformation, arteriovenous fistula, evacuation of hematomas, and decompressive craniotomy $(n=230)$, those with septic shock $(n=3)$, and those who received preoperative transfusion $(n=8)$. The last two categories were excluded because they were deemed to form an exceptionally morbid subgroup. After these exclusions, 721 patients formed the final study population (figure 1). 
Figure 1 Patient selection criteria. AVF, arteriovenous fistula; AVM, arteriovenous malformation; CPT, Current Procedural Terminology; ICD-9, international classification of diseases. ${ }^{*}$ CPT codes included in final analysis: $61584,61595,61597,61598,61700$, 61702, 61697-61698, 61703, 61705, 61711.

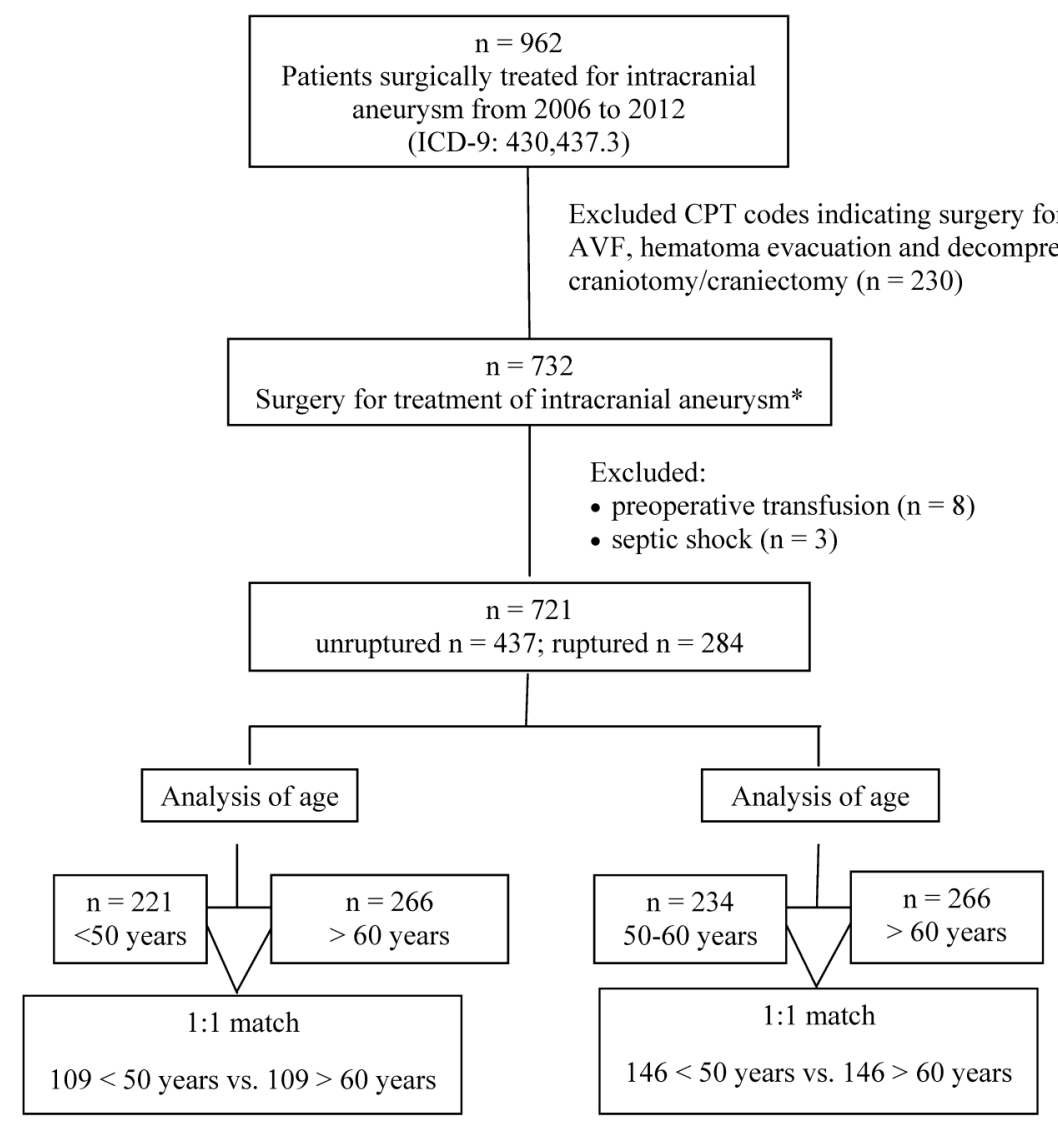

Patients were divided into three subgroups according to age at the time of surgery: $<50$ years $(n=221), 50-60$ years $(n=234)$, and $>60$ years $(n=266)$. These age groups were chosen because the age distribution of patients in the study group followed a normal Bell's distribution such that patients in the above age categories formed three distinct groups with similar number of subjects in each comprising almost 33\% of the entire population.

\section{Determination of Hunt and Hess Grade in NSQIP}

Although NSQIP does include Hunt and Hess $(\mathrm{HH})$ grade, we were able to approximate this for all patients with a ruptured aneurysm (ICD-9 code 430) based on the preoperative variable recorded in the database. Patients who were "unconscious, or postured to painful stimuli, or were unresponsive to all stimuli entering surgery" were captured by NSQIP under the variable 'coma'; we assigned these patients an $\mathrm{HH}$ grade of 5. Patients were considered to have 'hemiplegia' in NSQIP if they "had sustained acute or chronic neuromuscular injury resulting in total or partial paralysis or paresis of one side of the body, or if they had hemiplegia/hemiparesis upon arrival at the operating room, or if they had hemiplegia or hemiparesis associated with a cerebrovascular accident/stroke". We considered these patients to have $\mathrm{HH}$ grade 4 . We considered patients to have an $\mathrm{HH}$ grade of 3 if they had 'impaired sensorium', which is defined in NSQIP as follows: patients who "were acutely confused and/or delirious and responded to verbal and/or mild tactile stimulation, or who were noted to have developed an impaired sensorium if they have mental status changes, and/or delirium in the context of the current illness." Patients who had chronic or longstanding mental status changes secondary to chronic mental illness or chronic dementing illnesses were not considered to have impaired sensorium. Unfortunately, NSQIP does not provide any data on headache or nuchal rigidity, making it impossible to distinguish between patients with $\mathrm{HH}$ grades of 1 and 2. Thus, we had to combine $\mathrm{HH}$ grades 1 and 2 into one category; all patients who were not classified as $\mathrm{HH}$ grade 5, 4, or 3 were in this category.

\section{Outcomes}

We analyzed these outcome measures after surgery: (i) prolonged length of hospital stay, defined as a length of stay longer than the third quartile $(>75 \%$ of the sample, which was 17 days); (ii) minor complications, which included superficial surgical site infection, urinary tract infection, deep venous thrombosis, or thrombophlebitis; (iii) major complications, which consisted of deep incision surgical site infection, organ or space surgical site infection, wound disruption, pneumonia, unplanned intubation, pulmonary embolism, $>48 \mathrm{~h}$ postoperative ventilator-assisted respiration, progressive renal insufficiency, acute renal failure, cerebrovascular accident with neurological deficit, coma of $>24 \mathrm{~h}$, peripheral nerve injury, cardiac arrest requiring cardiopulmonary resuscitation, myocardial infarction, graft, prosthesis or flap failure, sepsis, septic shock, and/or 30-day return to the operation room; (iv) any complication, which was defined as having at least one minor or major complications; (v) unexpected return to the operation room, which was defined as a return for any major surgical procedure; (vi) discharged with continued care, which was defined as discharge to a care facility (exempting those who were initially admitted from such facilities); (vii) death within 30 days of index surgery.

\section{Statistical analysis}

We compared patients who were $>60$ years old with those who were aged $<50$ or 50-60 years. Owing to the retrospective 
nature of the analysis, this study is inherently prone to selection bias. In addition, adequate control for baseline characteristics is required to elucidate the impact of age as a potential independent risk factor for poor surgical outcome. ${ }^{17}$ We used propensity score matching to minimize selection bias by increasing the balance in baseline characteristics between the age groups. This is the closest approximation in a retrospective study to a randomized trial, ${ }^{18}$ and the methodology has been used previously in similarly designed studies. ${ }^{17}{ }^{19-22}$ A propensity score, in the context of this study, is the probability of a patient being grouped in a specific age group according to a set of observed covariates. ${ }^{23}$ The score is determined using multivariate logistic regression with each age group as a dependent variable and a set of covariates as independent variables. ${ }^{20}$ We included body mass index, race, smoking status, hypertension, diabetes, preoperative sepsis, perioperative transfusion, cerebrovascular comorbidities, American Association of Anesthesiologists class, and rupture status of the aneurysm as independent variables in the model. Once propensity scores were generated as described above, a 1:1 greedy matching technique was used to match patients in age group $<50$ and 50-60 with those aged $>60$. $^{24}$ We also determined the impact of age as a continuous variable, as 5 -year increments above age 50 , on the outcome of aneurysmal surgery.

We used Pearson's $\chi^{2}$ test and analysis of variance to compare categorical and continuous variables, respectively. A p value $<0.05$ was considered statistically significant. Covariate balance was determined using standardized difference before and after matching. A value of $>0.20$ was considered to denote significant covariate imbalance. ${ }^{18}$ Logistic regression reported as OR and 95\% CI was used to examine the association between increased age and adverse outcomes in the unmatched cohort. We used conditional logistic regression in the matched cohort in order to take into account the matched nature of data. We used SAS (V.9.2, SAS Institute) for all statistical analyses.

\section{RESULTS}

Table 1 shows the baseline characteristics, including demographic variables, abnormal preoperative laboratory values and comorbidities, among patients from each age group. Smoking status, excessive daily alcohol consumption, hypertension, diabetes, preoperative sepsis, abnormal preoperative white blood cell and liver function test, perioperative transfusion, and $\mathrm{HH}$ grade were the unbalanced covariates between the age groups.

In table 2, the baseline characteristics of each age group after propensity score matching are shown. We matched 109 subjects $(48 \%)$ to compare patients $>60$ and $<50$ years old, and 146 subjects $(55 \%)$ to compare patients $>60$ and 50-60 years old. Upon matching, we achieved covariate balance for all variables included in the analysis, indicating that propensity score matching was successful.

Postoperative complications were significantly more frequent among patients aged $>60(41.1 \%)$ than those $<50(25.3 \%)$ and $50-60(29.5 \%)$ years old, $\mathrm{p}<0.01$ for both (see online supplementary table S1). This observation persisted in the matched cohort. In the unmatched cohort, patients $>60$ years in comparison with those aged $<50$ were more likely to require continued care upon discharge $(34.0 \%$ vs $19.3 \%, \mathrm{p}=0.01)$ and die within 30 days of index surgery $(8.3 \%$ vs $3.2 \%, p=0.02)$. In the matched cohort, requirement for continued care persisted in patients aged $>60(44.9 \%$ vs $21.7 \%, \mathrm{p}<0.02)$.

In the general cohort, age $<50$ was associated with fewer postoperative complications $(\mathrm{OR}=0.5,95 \% \mathrm{CI} 0.3$ to 0.7$)$, continued care $(\mathrm{OR}=0.5,95 \% \mathrm{CI} 0.3$ to 0.8$)$, and 30 -day mortality
$(\mathrm{OR}=0.4,95 \%$ CI 0.2 to 0.9$)$ compared with those aged $>60$ (table 3). In the matched cohort, however, only postoperative complications $(\mathrm{OR}=0.4,95 \% \mathrm{CI} 0.2$ to 0.7$)$ were less likely to occur in patients aged $<50$. Similarly, postoperative complications were less likely in patients aged 50-60 years before $(\mathrm{OR}=0.6,95 \% \mathrm{CI} 0.4$ to 0.8$)$ and after $(\mathrm{OR}=0.5,95 \%$ CI 0.3 to 0.8 ) matching in comparison with those aged $>60$. Therefore, age $>60$ was independently associated with a more morbid 30-day postoperative course.

Sensitivity analyses using age as continuous variable showed that each additional 5 years above age 50 resulted in a slightly increased risk of postoperative complications $(\mathrm{OR}=1.16,95 \%$ CI 1.04 to 1.29 ), but not other adverse events.

\section{DISCUSSION}

Although other studies have examined the impact of age on the outcome of ICA management, this study is the first to provide a comprehensive analysis of the impact of age on surgical outcome for treatment of ICA, with meticulous adjustment of numerous demographic variables, preoperative laboratory values, and comorbidities. Our analysis showed that after matching for baseline characteristics, patients who were $>60$ years old had a higher risk of 30-day postoperative complications than those who were aged $50-60$ or $<50$ years.

\section{Past analyses of age as a risk factor in aneurysm treatment}

The incidence of unruptured ICA increases with age. Stehbens reported the highest incidence in patients aged 40 and $70 .^{25}$ Inagawa and Hirano reported peak incidence of unruptured ICA in patients aged $60-69 .^{12}$ In a recent meta-analysis of 68 studies, Vlak and colleagues reported that the prevalence of unruptured ICA was higher in patients aged $\geq 80$ years $(3.0 \%)$ than in those aged $\leq 30$ years, but no higher in patients in other age groups. $^{26}$

Age has been studied extensively as a determinant of the natural course of unruptured ICA. In the retrospective part of the International Study of Unruptured Intracranial Aneurysms (ISUIA), increased age was associated with a risk of rupture in patients with a history of treated aneurysmal SAH. ${ }^{27}$ In contrast, in a long-term follow-up of 142 patients, Juvela and colleagues found that the risk of aneurysmal SAH in patients diagnosed with unruptured ICA is inversely related to age. ${ }^{28}$ Similarly, the risk of rupture in patients with small ICAs ( $\leq 7 \mathrm{~mm}$ diameter) is also reported to be inversely related to age. ${ }^{29}$ In a retrospective study of 20767 Medicare patients, with an average age of 73.8 years, Taylor and colleagues reported that increased age is a protective factor against rupture of previously diagnosed ICA. ${ }^{30}$ In a recent Japanese study of 6697 patients with unruptured ICAs, an age of $\geq 70$ years was not associated with a risk of rupture. $^{31}$

The impact of age on the surgical outcome of ICA treatment is controversial, with numerous studies arguing for or against aggressive management in elderly patients. In the seminal report by ISUIA in 1998, surgery-related 1-year morbidity and mortality in patients $<45$ years of age was $6.5 \%$, compared with $14.4 \%$ for those aged $45-64$ years and 32\% for those aged $>64 .{ }^{27}$ However, baseline characteristics were not compared or adjusted for according to age strata. This limitation is present in another well-cited study by the International Cooperative Study on the Timing of Aneurysm Surgery. ${ }^{7} 32$ In this analysis, authors reported a favorable outcome in $90 \%$ of patients aged 18-29 with a $3 \%$ mortality rate compared with $56 \%$ good results and $20 \%$ mortality rate in patients aged $60-69 .{ }^{32}$ Kassell and colleagues used a multivariate regression model and found that 
Table 1 Baseline characteristics of patients undergoing surgery for intracranial aneurysm in the unmatched cohorts

\begin{tabular}{|c|c|c|c|c|c|c|c|}
\hline \multirow[b]{2}{*}{ Characteristics } & \multicolumn{3}{|l|}{ Age groups } & \multicolumn{2}{|c|}{$>60$ vs $<50$} & \multicolumn{2}{|c|}{$>60$ vs $50-60$} \\
\hline & $\begin{array}{l}>60 \text { Years } \\
(n=266)(\%)\end{array}$ & $\begin{array}{l}<50 \text { Years } \\
(n=221)(\%)\end{array}$ & $\begin{array}{l}50-60 \text { Years } \\
(n=234)(\%)\end{array}$ & p Value* & $\begin{array}{l}\text { Absolute } \\
\text { standardized } \\
\text { difference } \neq\end{array}$ & p Value* & $\begin{array}{l}\text { Absolute } \\
\text { standardized } \\
\text { difference } ¥\end{array}$ \\
\hline Female & 74.1 & 66.5 & 73.5 & 0.07 & 0.17 & 0.89 & 0.01 \\
\hline Caucasian & 69.0 & 60.6 & 64.8 & 0.06 & 0.18 & 0.34 & 0.09 \\
\hline Admitted from home & 73.3 & 73.3 & 69.2 & 1.0 & 0.00 & 0.31 & 0.09 \\
\hline \multicolumn{8}{|l|}{ Smoking status } \\
\hline Current & 28.8 & 50.0 & 51.1 & $<0.01$ & 0.49 & $<0.01$ & 0.47 \\
\hline Previous & 15.2 & 6.1 & 9.9 & & & & \\
\hline$>2$ Drinks a day & 2.2 & 7.0 & 7.1 & 0.02 & 0.23 & 0.02 & 0.23 \\
\hline Dependent functional status & 15.0 & 13.1 & 13.8 & 0.55 & 0.06 & 0.69 & 0.04 \\
\hline \multicolumn{8}{|l|}{ ASA classification } \\
\hline $1+2$ & 16.5 & 21.3 & 14.1 & 0.40 & 0.12 & 0.48 & 0.11 \\
\hline $3+4$ & 82.7 & 77.8 & 84.2 & & & & \\
\hline $\mathrm{BMI}, \mathrm{kg} / \mathrm{m}^{2}$, mean $\pm \mathrm{SD}$ & $28 \pm 6$ & $29 \pm 7$ & $28 \pm 7$ & 0.29 & 0.15 & 0.74 & 0.07 \\
\hline Cardiopulmonary comorbidities & 29.3 & 19.9 & 26.9 & 0.02 & 0.13 & 0.55 & 0.05 \\
\hline Diabetes mellitus & 14.7 & 3.2 & 13.7 & $<0.01$ & 0.41 & 0.75 & 0.03 \\
\hline Hypertension requiring medication & 68.4 & 37.1 & 52.1 & $<0.01$ & 0.67 & $<0.01$ & 0.34 \\
\hline Cerebrovascular comorbidities & 17.0 & 18.2 & 15.7 & 0.75 & 0.03 & 0.73 & 0.03 \\
\hline Renal comorbidities & 25.6 & 23.1 & 19.7 & 0.11 & 0.15 & 0.52 & 0.14 \\
\hline Cancer-related comorbidities & 1.5 & 0.5 & 0.9 & 0.25 & 0.10 & 0.51 & 0.06 \\
\hline Steroid use for chronic condition & 2.6 & 1.4 & 0.9 & 0.32 & 0.09 & 0.14 & 0.14 \\
\hline Preoperative SIRS or sepsis & 3.8 & 10.0 & 10.3 & 0.01 & 0.25 & $<0.01$ & 0.26 \\
\hline Bleeding risk factors & 12.4 & 9.5 & 8.6 & 0.31 & 0.05 & 0.31 & 0.13 \\
\hline Prior operation within 30 days & 1.4 & 4.3 & 0.5 & 0.07 & 0.18 & 0.37 & 0.09 \\
\hline Preoperative anemia & 38.4 & 29.4 & 28.2 & 0.04 & 0.05 & 0.02 & 0.07 \\
\hline Abnormal LFT & 12.8 & 14.9 & 12.0 & 0.49 & 0.18 & 0.78 & 0.02 \\
\hline Abnormal WBC & 26.1 & 37.1 & 28.6 & 0.01 & 0.24 & 0.54 & 0.06 \\
\hline Emergency & 26.7 & 27.2 & 26.9 & 0.91 & 0.01 & 0.95 & 0.01 \\
\hline Resident in operating room & 81.3 & 83.7 & 83.3 & 0.53 & 0.06 & 0.59 & 0.05 \\
\hline Intra- and postoperative transfusion & 20.7 & 7.2 & 12.0 & $<0.01$ & 0.40 & 0.01 & 0.24 \\
\hline Ruptured aneurysm & 35.0 & 44.3 & 39.7 & 0.04 & 0.19 & 0.27 & 0.10 \\
\hline \multicolumn{8}{|l|}{ Hunt and Hess gradet } \\
\hline 1 and 2 & 63.4 & 71.4 & 65.6 & 0.61 & 0.20 & 0.66 & 0.19 \\
\hline 3 & 21.5 & 18.4 & 15.1 & & & & \\
\hline 4 & 7.5 & 4.1 & 9.7 & & & & \\
\hline 5 & 7.5 & 6.1 & 9.7 & & & & \\
\hline
\end{tabular}

increased age was a prognostic factor for poor operative results, but comorbidities were not controlled. ${ }^{32}$ In another study by Wiebers et al, a multivariate regression model showed an age of $\geq 50$ years to be a multiplier of risk for poor surgical outcome in patients with unruptured ICA compared with those younger than $50 .{ }^{33}$ However, it is not clear which variables other than age were included in the model, raising concern as to whether adjustment for comorbidities was adequate. It is important to note that these studies were primarily designed to identify potential risk factors for poor outcomes in patients undergoing surgical management of ICA. This methodological approach justifies the use of multivariate regression, whereby one can determine the impact of multiple variables on the outcome of interest at once. However, to confirm the impact of a variable as complex as age, which itself is often associated with multiple comorbidities independently affecting surgical outcomes, appropriate adjustment of confounding factors is of paramount importance. ${ }^{17}$

\section{Studies using the Nationwide Inpatient Sample}

The impact of age on the outcome of surgical management of ICA has been studied using the Nationwide Inpatient Sample (NIS). ${ }^{3} 49$ In a study of more than 14000 patients from the NIS, Lawson and colleagues found that increasing age, as a continuous variable, is a modest predictor of poor outcome for clipping ICAs $(\mathrm{OR}=1.07,95 \%$ CI 1.055 to 1.075$) .{ }^{9}$ A descriptive analysis of the following medical comorbidities was provided in the study: alcoholism, heart failure, chronic lung disease, chronic hypertension, diabetes, hepatic disease, coagulopathy, drug abuse, obesity, and renal failure. However, it is not clear which variables were included in the logistic regression. Brinjikji and colleagues studied nearly 30000 patients who underwent clipping of a cerebral aneurysm and found, in a multivariable logistic regression, that increased age was a risk factor for discharge to long-term facility and mortality. ${ }^{4}$ Barker and colleagues, using the NIS, compared the impact of age on the outcome of coiling with clipping ICAs. ${ }^{3}$ The authors found 
Table 2 Baseline characteristics of patients $>60$ and 50-60 years of age undergoing surgery for intracranial aneurysm in the matched cohort

\begin{tabular}{|c|c|c|c|c|c|c|c|c|}
\hline \multirow[b]{2}{*}{ Characteristics } & \multicolumn{4}{|l|}{$>60$ vs $<50$} & \multicolumn{4}{|l|}{$>60$ vs $50-60$} \\
\hline & $\begin{array}{l}>60 \text { Years } \\
(n=109)(\%)\end{array}$ & $\begin{array}{l}<50 \text { Years } \\
(n=109)(\%)\end{array}$ & $p$ Value & $\begin{array}{l}\text { Absolute } \\
\text { standardized } \\
\text { difference }\end{array}$ & $\begin{array}{l}>60 \text { Years } \\
(n=146)(\%)\end{array}$ & $\begin{array}{l}50-60 \\
\text { Years } \\
(n=146)(\%)\end{array}$ & $p$ Value & $\begin{array}{l}\text { Absolute } \\
\text { standardized } \\
\text { difference }\end{array}$ \\
\hline Female & 75.2 & 70.6 & 0.45 & 0.10 & 74.0 & 76.7 & 0.59 & 0.06 \\
\hline Caucasian & 64.2 & 65.1 & 0.89 & 0.02 & 69.6 & 66.7 & 0.61 & 0.06 \\
\hline Admitted from home & 71.6 & 76.2 & 0.44 & 0.10 & 74.0 & 72.6 & 0.79 & 0.03 \\
\hline \multicolumn{9}{|l|}{ Smoking status } \\
\hline Current & 39.5 & 43.1 & 0.86 & 0.08 & 41.1 & 48.6 & 0.43 & 0.15 \\
\hline Previous & 9.2 & 8.3 & & & 15.1 & 13.0 & & \\
\hline$>2$ Drinks a day & 3.7 & 5.5 & 0.52 & 0.09 & 3.4 & 5.5 & 0.39 & 0.10 \\
\hline Dependent functional status & 19.3 & 16.5 & 0.60 & 0.07 & 17.8 & 13.7 & 0.34 & 0.11 \\
\hline \multicolumn{9}{|l|}{ ASA classification } \\
\hline $1+2$ & 18.4 & 20.2 & 0.94 & 0.05 & 13.7 & 16.4 & 0.81 & 0.08 \\
\hline $3+4$ & 80.7 & 78.9 & & & 84.9 & 82.2 & & \\
\hline $\mathrm{BMI}, \mathrm{kg} / \mathrm{m}^{2}$, mean $\pm \mathrm{SD}$ & $29 \pm 7$ & $29 \pm 8$ & 0.52 & 0.01 & $29 \pm 7$ & $28 \pm 7$ & 0.34 & 0.08 \\
\hline Cardiopulmonary comorbidities & 20.2 & 13.8 & 0.21 & 0.17 & 28.8 & 25.3 & 0.51 & 0.11 \\
\hline Diabetes mellitus & 4.6 & 4.6 & 1.0 & 0.00 & 15.8 & 15.1 & 0.87 & 0.02 \\
\hline Hypertension requiring medication & 54.1 & 49.5 & 0.50 & 0.09 & 58.9 & 54.1 & 0.41 & 0.10 \\
\hline Cerebrovascular comorbidities & 16.5 & 19.3 & 0.60 & 0.07 & 15.8 & 15.1 & 0.87 & 0.02 \\
\hline Renal comorbidities & 0.9 & 0.0 & 0.32 & 0.14 & 16.7 & 15.1 & 0.01 & 0.09 \\
\hline Cancer-related comorbidities & 1.8 & 0.9 & 0.56 & 0.08 & 2.1 & 0.7 & 0.31 & 0.12 \\
\hline Steroid use for chronic condition & 1.8 & 1.8 & 1.0 & 0.00 & 2.1 & 0.7 & 0.31 & 0.12 \\
\hline Preoperative SIRS or sepsis & 5.5 & 8.3 & 0.42 & 0.11 & 4.8 & 6.2 & 0.61 & 0.06 \\
\hline Bleeding risk factors & 3.8 & 4.9 & 0.70 & 0.05 & 11.6 & 9.6 & 0.57 & 0.08 \\
\hline Prior operation within 30 days & 0.9 & 0.9 & 1.0 & 0.00 & 0.7 & 0.7 & 1.0 & 0.00 \\
\hline Preoperative anemia & 29.4 & 31.2 & 0.77 & 0.05 & 31.5 & 27.4 & 0.44 & 0.08 \\
\hline Abnormal LFT & 14.6 & 14.6 & 1.0 & 0.00 & 13.0 & 9.6 & 0.59 & 0.10 \\
\hline Abnormal WBC & 30.1 & 31.4 & 0.84 & 0.03 & 27.0 & 28.1 & 0.85 & 0.02 \\
\hline Emergency & 24.8 & 25.7 & 0.88 & 0.02 & 25.3 & 19.2 & 0.21 & 0.15 \\
\hline Resident in operating room & 79.8 & 82.6 & 0.60 & 0.07 & 81.5 & 82.2 & 0.88 & 0.02 \\
\hline Intra- and postoperative transfusion & 10.1 & 9.2 & 0.82 & 0.03 & 14.4 & 13.0 & 0.73 & 0.04 \\
\hline Ruptured aneurysm & 39.5 & 41.3 & 0.78 & 0.04 & 34.3 & 34.9 & 0.90 & 0.01 \\
\hline \multicolumn{9}{|l|}{ Hunt and Hess gradet } \\
\hline 1 and 2 & 63.4 & 71.4 & 0.61 & 0.19 & 52.0 & 58.8 & 0.89 & 0.16 \\
\hline 3 & 21.5 & 18.4 & & & 28.0 & 21.6 & & \\
\hline 4 & 7.5 & 4.1 & & & 8.0 & 7.8 & & \\
\hline 5 & 7.5 & 6.1 & & & 12.0 & 11.8 & & \\
\hline
\end{tabular}

ASA, American Association of Anesthesiologists; BMI, body mass index; LFT, liver function test; SIRS, systemic inflammatory response syndrome; WBC, white blood cell.

that, at age $>65$, the difference between clipping and coiling becomes statistically significant, but outcome of clipping among different age groups was not compared explicitly. In our analysis, we did not study patients who underwent coiling.

The above-mentioned studies benefited from a large number of subjects, thus increasing the power of analysis. However, adequate control of medical comorbidities was unclear or not undertaken or failed to add statistical power. ${ }^{349}$ The lack of control for comorbidities significantly limits the power of these studies and may severely exaggerate the impact of these comorbidities because they are more common in an older population. Moreover, the NIS is an administrative database constructed retrospectively and based on admission records and billing information, and the validity of an administrative database use for the study of ICA has been questioned. ${ }^{18}$ Alternatively, NSQIP is a clinical database constructed prospectively with proven validity and reproducibility. ${ }^{15}{ }^{16}$ Thus, our analysis, although smaller, presents a robust methodology using a national prospective clinical database to separate age from medical comorbidities using propensity scores.
Adjustment for comorbidities in analysis of age

The assumption that risk related to comorbidities increases with advanced age is not necessarily true. In a retrospective study of 219 patients who underwent craniotomy for ICA, Stachniak and colleagues reported a similar number of complications and duration of hospitalization for patients $\geq 65$ and $<65$ years old, despite poor APACHE II (Acute Physiology and Chronic Health Evaluation) scores in the former. ${ }^{11}$ The authors concluded that patients older than 65 can benefit from surgery for treatment of ICA, ruptured or unruptured. ${ }^{11}$ In a study from Sweden, ${ }^{34}$ two-thirds of 76 patients aged $70-74$ had good surgical outcome for the treatment of ICA, but younger age groups were not used as control and patients with severe cardiovascular or cerebrovascular disease were excluded. In a single-institution descriptive study of 129 consecutive patients aged $\geq 70$ years with ruptured or unruptured ICA, Chung and colleagues reported good 6-month management outcomes in patients with unruptured aneurysms, but no control group was used. ${ }^{5}$ Overall, the findings of these studies suggest that increased age alone should not be a contraindication to surgery. 
Table 3 Postoperative complications

\begin{tabular}{|c|c|c|c|c|}
\hline \multirow[b]{3}{*}{ Adverse outcomes } & \multicolumn{4}{|c|}{ Odds ratio $(95 \% \mathrm{Cl})^{*}$} \\
\hline & \multicolumn{2}{|c|}{$<50$ Years vs $>60$ years } & \multicolumn{2}{|c|}{$50-60$ Years vs $>60$ years } \\
\hline & General cohortt & Matched cohort $\ddagger$ & General cohortt & Matched cohort‡ \\
\hline Prolonged LOS (>17 days) & $0.7(0.5$ to 1.1$)$ & 0.7 (0.4 to 1.3$)$ & $0.9(0.6$ to 1.4$)$ & $0.8(0.5$ to 1.4$)$ \\
\hline Minor complications & $0.6(0.3$ to 1.1$)$ & $0.5(0.2$ to 1.2$)$ & $0.5(0.3$ to 0.9$)$ & $0.4(0.2$ to 1.0$)$ \\
\hline Major complications & $0.5(0.3$ to 0.7$)$ & $0.3(0.2$ to 0.7$)$ & $0.6(0.4$ to 0.9$)$ & $0.5(0.3$ to 0.9$)$ \\
\hline Any complications & $0.5(0.3$ to 0.7$)$ & $0.4(0.2$ to 0.7$)$ & $0.6(0.4$ to 0.8$)$ & $0.5(0.3$ to 0.8$)$ \\
\hline 30 -Day return to the operating room & $0.8(0.5$ to 1.4$)$ & $1.1(0.5$ to 2.7$)$ & $0.6(0.3$ to 1.1$)$ & $0.8(0.4$ to 1.7$)$ \\
\hline Required continued care & $0.5(0.3$ to 0.8$)$ & N/A & $0.8(0.5$ to 1.4$)$ & $0.5(0.2$ to 1.2$)$ \\
\hline 30-Day mortality & $0.4(0.2$ to 0.9$)$ & $0.2(0.0$ to 1.4$)$ & 0.7 (0.4 to 1.4$)$ & $1.1(0.4$ to3.2) \\
\hline
\end{tabular}

Age as an independent risk factor for poor surgical outcome must be separated from comorbidities that often become prevalent with increased age. ${ }^{8} 1117$ In our analysis, patients in older age groups had a higher frequency of comorbid conditions, abnormal laboratory values, and perioperative transfusion, all of which might confound the impact of age on the outcome of surgical management of ICA, and which may explain, at least in part, the decreased risk of a morbid postoperative course in patients 50-60 years old compared with those $>60$ years old. Furthermore, in this series the risk of death within 30 days of surgery was lower in patients aged $<50$. We used propensity score matching to adjust for baseline characteristics. This methodology has been used in other neurosurgical outcome studies. ${ }^{17}$ 19-21 Thereafter, age >60 independently incurred increased risk of postoperative complications compared with younger age groups, but not for mortality. Thus discrepancy in distribution of comorbidities among patients in different age groups may have a drastic effect in analysis.

\section{Clinical implications}

In this study, we found that patients aged $>60$ are at increased risk of perioperative complications after cerebral aneurysm surgery. Consideration of age as a categorical variable may hinder applicability of the findings to neurosurgical practice. Using age as continuous variable, each 5 -year interval increases the risk of perioperative complications, confirming that the association between age $>60$ and complications, as revealed in categorical analyses, is not a sudden change in risk but a trend, suggesting that increased age gradually confers morbidity in cerebral aneurysm surgery. Furthermore, this is not to say that all patients aged $>60$ fare the same; within this age group too, patients will differ depending on specific risk factors. In addition, age is not the only risk factor to consider when evaluating a patient as potential candidate for surgery. Other variables include aneurysm location, morphology, and amenability to endovascular treatment. But this study encourages neurosurgeons to consider increased age as a potential independent risk factor for compromised outcome of open surgery for aneurysm, when all other variables mentioned above, and otherwise, are equal.

\section{Limitations}

This study has some limitations. Query of the database for Current Procedural Terminology codes for endovascular treatment of cerebral aneurysm (61710) found 17 patients during the study period
2006-2012. This small number might be attributed to the fact that (1) these cases do not constitute a sufficiently large proportion of neurosurgical procedures as a whole, and hence are not often captured in ACS-NSQIP, which randomly selects patients within the surgical department at each participating institution and (2) ACS-NSQIP does not contain data from radiological or neurological endovascular cases. With gradual increase in the prevalence of coiling for treatment of ICA, we suspect that patients who underwent open surgery in later years might have had a more complex aneurysm. Thus, the complexity of disease in our cohort of more than 700 patients might not be homogeneous. NSQIP, a surgical database, contains data for patients who have had an operation; hence, patients who had been denied surgery on the basis of age only would not have been included in NSQIP. Using propensity score matching, we controlled for all available demographic variables, preoperative laboratory values, and comorbidities. Although this methodology allowed us to fully balance the distribution of available covariates, any unknown or unavailable covariates might confound our analyses-an inherent limitation of any retrospective study. Therefore, we cannot confirm nor refute a cause and effect relationship between age $>60$ and poor surgical outcome. Of note, we do not have access to some aneurysmspecific variables. These are invariably considered in management and are well-established prognostic factors. ${ }^{27}$ We cannot discern in ACS-NSQIP whether patients were treated at centers where endovascular treatment was available and if it were, whether the patient underwent endovascular treatment before surgery. However, the variable 'prior operation within 30 days' does capture endovascular treatment, and as demonstrated in table $1,<5 \%$ of patients in each age group underwent prior surgery within 30 days. As a short-term study, we are unable to extrapolate the findings of this study beyond 30 days postoperatively. Despite these limitations, the data in this study are from a prospectively collected database from 372 academic and non-academic hospitals in the USA with standardized and validated data collection, ${ }^{16}$ and we attempted to deal with the methodological limitations of previous studies, some of which report similar findings.

\section{CONCLUSIONS}

Although the impact of age on the outcome of surgery for aneurysm has been studied before, this study is unique in its study design, rigor of statistical analysis, and quality of the database. Using a nationwide prospectively collected database, we evaluated the impact of increased age on 30-day morbidity and mortality in patients undergoing surgery for management of 
ruptured and unruptured ICAs. After propensity score matching, age $>60$, as a categorical variable, was associated with postoperative complications. This study suggests that in surgery for management of cerebral aneurysms, age $>60$ should be considered an a priori risk factor independent of any other comorbidities for short-term outcome of open surgery.

Contributors NA: conception, interpretation of results, drafting, critical revision, and final approval of the manuscript. AS: conception, acquisition and analysis of data, interpretation of results, critical revision, and final approval of the manuscript. SS: acquisition and analysis of data, final approval of manuscript. WRS: conception, critical revision and final approval of the manuscript. NCB: conception, critical revision, final approval of the manuscript, and study supervision.

\section{Competing interests None.}

Provenance and peer review Not commissioned; externally peer reviewed.

\section{REFERENCES}

1 Menghini VV, Brown RD Jr, Sicks JD, et al. Incidence and prevalence of intracranial aneurysms and hemorrhage in Olmsted County, Minnesota, 1965 to 1995. Neurology 1998;51:405-11.

2 Seibert B, Tummala RP, Chow R, et al. Intracranial aneurysms: review of current treatment options and outcomes. Front Neurol 2011;2:45.

3 Barker FG II, Amin-Hanjani S, Butler WE, et al. Age dependent differences in short-term outcome after surgical or endovascular treatment of unruptured intracranial aneurysms in the United States, 1996-2000. Neurosurgery 2004;54:18-28.

4 Brinjikji W, Rabinstein AA, Lanzino G, et al. Effect of age on National Inpatient Sample 2001-2008. Stroke 2013;42:1320-4.

5 Chung RY, Carter BS, Norbash A, et al. Management outcomes for ruptured and unruptured aneurysms in the elderly. Neurosurgery 2000;47:827-32.

6 Inagawa T, Yamamoto M, Kamiya K, et al. Management of elderly patients with aneurysmal subarachnoid hemorrhage. J Neurosurg 1988;69:332-9.

7 Kassell NF, Torner JC, Haley EC Jr, et al The international cooperative study on the timing of aneurysm surgery. Part 1: Overall management results. J Neurosurg 1990;73:18-36.

8 Lanzino G, Kassell NF, Germanson TP, et al. Age and outcome after aneurysmal subarachnoid hemorrhage: why do older patients fare worse? I Neurosurg 1996:85:410-8.

9 Lawson MF, Neal DW, Mocco J, et al. Rationale for treating unruptured intracranial aneurysms: actuarial analysis of natural history risk versus treatment risk for coiling or clipping based on 14,050 patients in the Nationwide Inpatient Sample database. World Neurosurg 2013;79:472-8.

10 Sakaki $S$, Ohta $S$, Ohue $S$, et al. Outcome in elderly patients with ruptured intracranial aneurysm. Clin Neurol Neurosurg 1989;91:21-7.

11 Stachniak JB, Layon AJ, Day AL, et al. Craniotomy for intracranial aneurysm and subarachnoid hemorrhage. Is course, cost, or outcome affected by age? Stroke 1996:27:276-81.

12 Inagawa T, Hirano A. Autopsy study of unruptured incidental intracranial aneurysms. Surg Neurol 1990;34:361-5.

13 American College of Surgeons National Surgical Quality Improvement Project. Inclusion/Exclusion Criteria, 2013. http://site.acsnsqip.org/programspecifics/ inclusionexclusion-criteria-4/ (accessed 16 Sep 2013).
14 Shiloach M, Frencher SK Jr, Steeger JE, et al. Toward robust information: data quality and inter-rater reliability in the American College of Surgeons National Surgical Quality Improvement Program. J Am Coll Surg 2010;210:6-16.

15 American College of Surgeons National Surgical Quality Improvement Project. User guide for the 2011 participant use data file. American College of Surgeons, October 2012.

16 Khuri SF, Henderson WG, Daley J, et al. The patient safety in surgery study: background, study design, and patient populations. J Am Coll Surg 2007;204:1089-102.

17 Seicean A, Seicean S, Schiltz NK, et al. Short-term outcomes of craniotomy for malignant brain tumors in the elderly. Cancer 2013;119:1058-64.

18 Stürmer T, Joshi M, Glynn RJ, et al. A review of the application of propensity score methods yielded increasing use, advantages in specific settings, but not substantially different estimates compared with conventional multivariable methods. J Clin Epidemiol 2006;59:437-47.

19 Lad SP, Bagley JH, Karikari IO, et al. Cancer after spinal fusion: the role of bone morphogenetic protein. Neurosurgery 2013;73:440-9.

20 Seicean A, Seicean S, Alan N, et al. Effect of smoking on the perioperative outcomes of patients who undergo elective spine surgery. Spine 2013;38:1294-302.

21 Seicean A, Seicean S, Alan N, et al. Preoperative anemia and perioperative outcomes in patients who undergo elective spine surgery. Spine 2013;38: 1331-41.

22 Alan N, Seicean A, Seicean S, et al. Impact of preoperative anemia on outcomes in patients undergoing elective cranial surgery. J Neurosurg 2014;120:764-72.

23 Rosenbaum PR, Rubin D. The central role of the propensity score in observational studies for causal effects. Biometrika 1983;70:41-55.

24 Bergstralh E, Kosanke J. Computerized matching of cases to controls. Technical Report Serial No. 56. Minnesota: Mayo Clinic Section of Biostatistics, 1995.

25 Stehbens BE. Aneurysms and anatomical variation of cerebral arteries. Arch Pathol 1963;75:45-64

26 Vlak MH, Algra A, Brandenburg R, et al. Prevalence of unruptured intracranial aneurysms, with emphasis on sex, age, comorbidity, country, and time period: a systematic review and meta-analysis. Lancet Neurol 2011;10:626-36.

27 International Study of Unruptured Intracranial Aneurysms Investigators. Unruptured intracranial aneurysms — risk of rupture and risks of surgical intervention. $N$ Engl J Med 1998:339:1725-33

28 Juvela S, Porras M, Poussa K. Natural history of unruptured intracranial aneurysms: probability of and risk factors for aneurysm rupture. J Neurosurg 2008;108:1052-60.

29 Nahed BV, DiLuna ML, Morgan T, et al. Hypertension, age, and location predict rupture of small intracranial aneurysms. Neurosurgery 2005;57:676-83.

30 Taylor CL, Yuan Z, Selman WR, et al. Cerebral arterial aneurysm formation and rupture in 20,767 elderly patients: hypertension and other risk factors. J Neurosurg 1995;83:812-9.

31 UCAS Japan Investigators. The natural course of unruptured cerebral aneurysms in a Japanese cohort. N Engl J Med 2012;366:2474-82.

32 Kassell NF, Torner JC, Jane JA, et al. The international cooperative study on the timing of aneurysm surgery. Part 2: Surgical results. J Neurosurg 1990;73:37-47.

33 Wiebers DO, Whisnant JP, Huston J III, et al Unruptured intracranial aneurysms: natural history, clinical outcome, and risks of surgical and endovascular treatment. Lancet 2003;362:103-10.

34 Fridriksson SM, Hillman J, Säveland $\mathrm{H}$, et al. Intracranial aneurysm surgery in the 8th and 9th decades of life: impact on population-based management outcome. Neurosurgery 1995;37:627-31. 